\title{
Neuronal polarization in the developing cerebral cortex
}

\author{
Akira Sakakibara ${ }^{1 *}$ and Yumiko Hatanaka ${ }^{2,3 *}$ \\ ${ }^{1}$ College of Life and Health Sciences, Chubu University, Kasugai, Japan, ${ }^{2}$ Division of Cerebral Circuitry, National Institute for \\ Physiological Sciences, Okazaki, Japan, ${ }^{3}$ Japan Science and Technology Agency, Core Research for Evolutional Science and \\ Technology, Tokyo, Japan
}

\section{OPEN ACCESS}

Edited by:

Chiaki Ohtaka-Maruyama,

Tokyo Metropolitan Institute of Medical

Science, Japan

Reviewed by:

Juan Andrés De Carlos,

Instituto Cajal (Consejo Superior de Investigaciones Cientificas), Spain

Anthony Paul Barnes,

Oregon Health and Science University,

${ }^{*}$ Correspondence:

Akira Sakakibara

College of Life and Health Sciences,

Chubu University, Kasugai, Aichi

487-8501, Japan

asakaki@isc.chubu.ac.jp;

Yumiko Hatanaka

Division of Cerebral Circuitry, National Institute for Physiological Sciences,

Higashiyama 5-1, Myodaiji, Okazaki,

Aichi 444-8787, Japan

yhatanaka-ns@umin.ac.jp

Specialty section:

This article was submitted to

Neurogenesis, a section of the journal

Frontiers in Neuroscience

Received: 29 January 2015 Paper pending published:

16 February 2015

Accepted: 22 March 2015

Published: 08 April 2015

Citation:

Sakakibara A and Hatanaka Y (2015)

Neuronal polarization in the

developing cerebral cortex.

Front. Neurosci. 9:116.

doi: 10.3389/fnins.2015.00116
Cortical neurons consist of excitatory projection neurons and inhibitory GABAergic interneurons, whose connections construct highly organized neuronal circuits that control higher order information processing. Recent progress in live imaging has allowed us to examine how these neurons differentiate during development in vivo or in in vivo-like conditions. These analyses have revealed how the initial steps of polarization, in which neurons establish an axon, occur. Interestingly, both excitatory and inhibitory cortical neurons establish neuronal polarity de novo by undergoing a multipolar stage reminiscent of the manner in which polarity formation occurs in hippocampal neurons in dissociated culture. In this review, we focus on polarity formation in cortical neurons and describe their typical morphology and dynamic behavior during the polarization period. We also discuss cellular and molecular mechanisms underlying polarization, with reference to polarity formation in dissociated hippocampal neurons in vitro.

Keywords: neuron, polarization, axon, cerebral cortex, imaging, excitatory cortical neuron, inhibitory cortical neuron

\section{Introduction}

Neurons are highly polarized cells that typically exhibit a single axon and several dendrites. Dendrites receive incoming signals at the synapse and convey them to the soma. These signals trigger action potentials at the level of soma, which propagate along the axon and are transmitted to target cells at a presynaptic site. A critical question in neurobiology is how neurons acquire axon-dendrite polarity, a property required for directional information flow in the nervous system.

Axon-dendrite polarization has been historically examined using cultured, dissociated hippocampal neurons (Dotti et al., 1988). These neurons initially appear symmetric, extending and retracting several immature neurites of similar length. Elongation of a single process, the one that will become the axon, breaks this symmetry. Thus, based on this model, neuronal polarity formation has been believed to result from a stochastic symmetry-breaking event. However, more recent morphological and imaging studies in vivo or in situ (in in vivo-like conditions that maintain an intact three-dimensional structure surrounding immature neurons) suggest that several types of neurons establish neuronal polarity by inheriting apicobasal polarity from neuroepithelial progenitors or maintaining front-rear polarity in migrating cells (Barnes and Polleux, 2009; Hatanaka et al., 2012). Therefore, it remained uncertain whether these activities occurred in vivo and, if so, whether they were regulated by similar events as that appear in cultured hippocampal neurons.

The cerebral cortex is evolutionary the youngest and the most complex region of the brain. It is composed primarily of excitatory neurons, which are glutamatergic, and by a smaller proportion of inhibitory neurons, which are GABA ( $\gamma$-aminobutyric acid)-ergic. During development, excitatory 
neurons originate from the dorsal telencephalon (Molyneaux et al., 2007), while inhibitory neurons originate from the ventral telencephalon (Gelman and Marin, 2010). Both subtypes are then integrated into the cerebral cortex and extend axons and dendrites to establish functional cortical circuitry. Interestingly, recent imaging studies have revealed how these dynamic developmental processes occur in vivo or in situ. Those studies suggest that most cortical neurons likely establish an axon via an initial symmetry-breaking event, a process similar to that observed in cultured hippocampal neurons.

In this review, we first give an overview of current knowledge about the developmental process of axon and dendrite formation of excitatory and inhibitory neurons in the cerebral cortex. Then we focus on the dynamic behavior underlying axon formation of these neurons, with reference to dissociated hippocampal neurons. Although there is evidence that some neurons may inherit some aspects of polarity emerged at a stage prior to axon formation, the model based on hippocampal cells still predominates in this field and could explain behavior of cortical neurons. We thus further summarize both intracellular signals and cytoskeletal dynamics underlying polarity formation in dissociated hippocampal neurons and in cortical neurons in vivo or in situ.

\section{Axon-Dendrite Polarization of Excitatory and Inhibitory Cortical Neurons}

The cerebral cortex is composed of the neocortex and allocortex. The neocortex, which is a six-layered structure unique to mammals, is phylogenetically the youngest brain region and comprises most of the cortex. In contrast, the allocortex is phylogenenetically older and characterized by fewer layers than the neocortex. The development of polarity by neocortical cells is the major focus of this review.

In rodents, cortical neurons are comprised of 70-80\% excitatory and $20-30 \%$ inhibitory neurons. Excitatory projecting neurons convey cortical output to subcortical structures and to other cortical areas. In general, neurons exhibiting corticofugal projections, which extend axons away from the cortex, reside in deep layers; by contrast, neurons that project intracortically extend axons to areas in the ipsilateral and/or contralateral cortex, reside in upper layers and to a lesser extent in deep layers (Greig et al., 2013). Depending on layer location and projection, excitatory neuron morphology varies. However, many excitatory neurons resemble so-called "pyramidal cells": their soma is shaped like a pyramid with a base facing the apical aspect of the cortex, and these cells extend a single axon and two separate apical and basal dendrites (Jones, 1984). Their axons extend toward the white matter (WM) where they typically turn and continue to project tangentially, while their apical dendrites extend toward the pial surface. Inhibitory neurons, on the other hand, are mostly local-circuit neurons that contribute to intracortical information processing by modulating excitability and thus shaping cortical output. Inhibitory neurons also extend a single axon and multiple dendrites, but their morphologies are highly diverse: they include basket cells, chandelier cells, Martinotti cells, double bouquet cells, neurogliaform cells, and at least 10 others (Kubota, 2014). Until now, however, only a few reports have described how axons or dendrites emerge from these neurons (e.g., Kawaguchi, 1993).

\section{Axon Formation Is the Initial Step of Cortical Neuronal Polarization}

Excitatory and inhibitory cortical neurons originate in distinct brain regions: the former emerge from the pallium and the latter primarily from the subpallium (Molyneaux et al., 2007; Gelman and Marin, 2010). Recent advances in cell labeling techniques, including use of genetically-modified mice and in utero electroporation methods, allow us to label these neurons accurately. Furthermore, advanced imaging techniques have revealed dynamic processes underlying their development.

\section{Development of Excitatory Cortical Neurons}

Excitatory cortical neurons originate predominantly from radial glial progenitors in the cortical ventricular zone (VZ) (Figure 1). Asymmetric division of these cells generates both self-renewing progenitors and young neurons or intermediate progenitors, and those intermediate progenitors then further divide to increase neuronal number (Miyata et al., 2001, 2004; Noctor et al., 2001, 2004; Pontious et al., 2008). Newly-generated neurons migrate through the subventricular zone (SVZ) and intermediate zone (IZ) to reach the cortical plate (CP). There, later-generated neurons migrate past neurons generated earlier and eventually occupy more superficial positions, resulting in an insidefirst/outside-last neurogenetic gradient (Angevine and Sidman, 1961). Following completion of this migration, these activities give rise to a sixed-layered cortical structure (Bayer and Altman, 1991).

Morphologically, bipolar progenitor cells are extraordinarily slender and extend a long apical process toward the pial surface and a short basal process toward the ventricle. During asymmetric cell division, the daughter cell destined to become a neuron assumes a multipolar shape from which emanates multiple short, thin processes in the SVZ/IZ (Tabata and Nakajima, 2003; Noctor et al., 2004). After repeated extension and retraction of processes over several hours, one process suddenly extends tangentially within the IZ (Hatanaka and Yamauchi, 2013; Namba et al., 2014; Sakakibara et al., 2014). That process continues to elongate and eventually becomes an axon, as indicated by its length, morphology, and accumulation axonal markers such as Kif5c560 in the tip (Hatanaka and Yamauchi, 2013; see also Section Intracellular Mechanisms). Immature axonal processes also contain bidirectional microtubule fibers (Sakakibara et al., 2014), and in this aspect exhibit the kind of mixed microtubule polarity typically seen in the trailing process of migrating cerebellar granule cells, which also becomes an axon (Rakic et al., 1996). The remaining short processes gradually transform into thick leading processes directed toward the pia (Hatanaka and Yamauchi, 2013; Sakakibara et al., 2014). By the time cell bodies reach the CP, they appear bipolar, extending a trailing process "behind" (opposite the direction of migration) and a leading process "in front" of the nucleus. Cells then migrate with glia-guided locomotion mode (Rakic, 1972; Nadarajah and Parnavelas, 2002) characterized by 


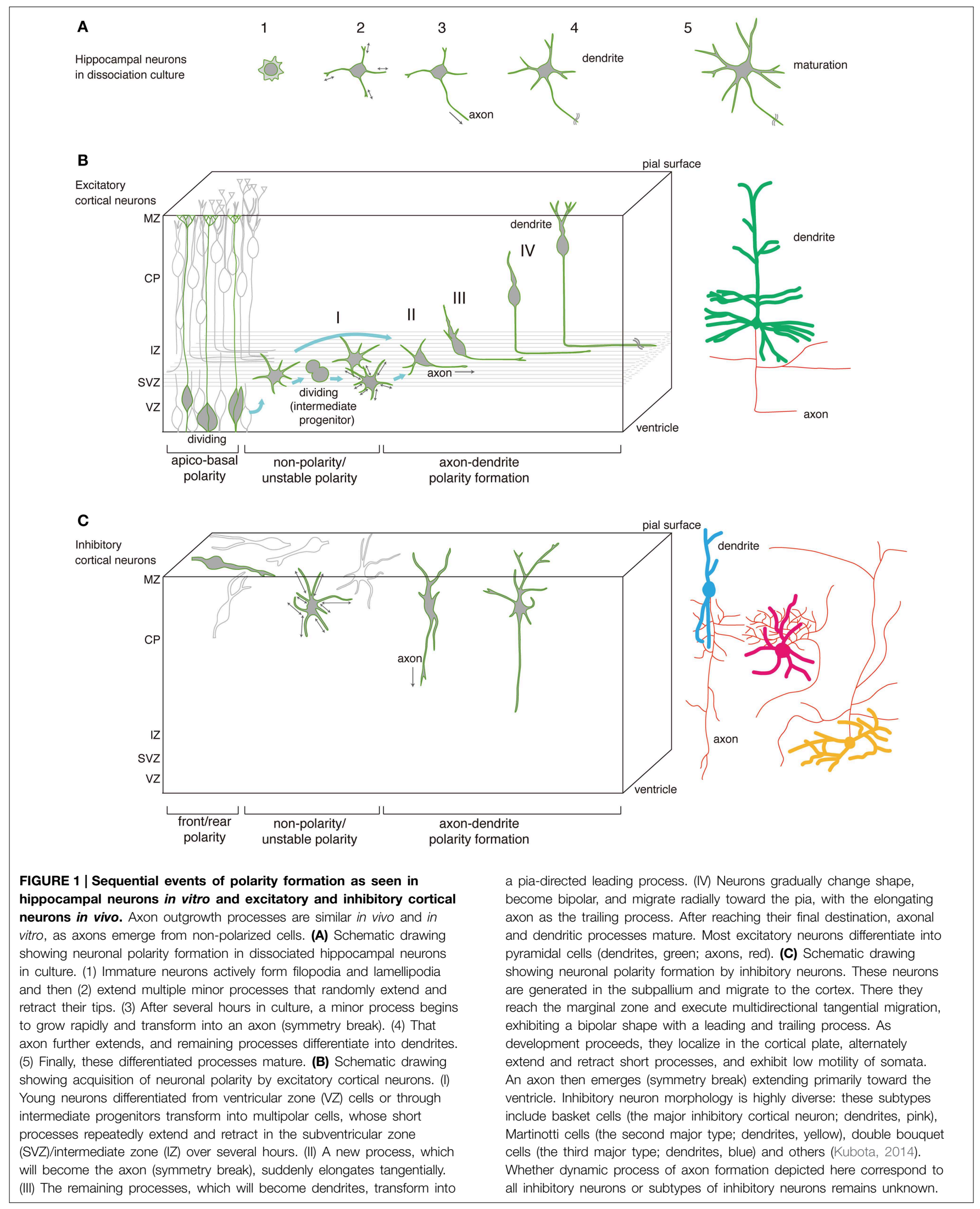


repeated short extensions and contractions of the leading process accompanied by saltatory cell movement. Once the leading process reaches the marginal zone (MZ), cells switch to somal translocation as their final mode of movement (Nadarajah and Parnavelas, 2002). The leading process then differentiates into a dendritic arbor-like structure (Hatanaka and Murakami, 2002), which probably develops into an apical dendrite. Since radial migration is accompanied by sustained trailing process elongation in the IZ (the future WM), this dynamic behavior eventually results in the typical pyramidal cell morphology in which an axon extends from the bottom of the soma toward the WM and an apical dendrite orients toward the pia. Thus, for excitatory cortical neurons, migration is closely related to establishment of prospective neuronal polarity.

\section{Development of Inhibitory Cortical Neurons}

Tangential migration of neurons that transgress the corticostriatal boundary and enter the neocortex was first reported by de Carlos et al. (1996). Collectively, several subsequent studies using transplantation, genetic fate mapping, and cell labeling analysis in vivo and in vitro established that tangentially migrating neurons include inhibitory cortical neurons. Moreover, most, if not all, inhibitory cortical neurons in mouse are reportedly generated embryonically from regions in the subpallium, including the medial and caudal ganglionic eminences and the preoptic area (Gelman and Marin, 2010), although some investigators have called into question whether cells emerge from the preoptic area (Ceci et al., 2012). Inhibitory neurons from these regions are further subdivided into distinct morphological subtypes exhibiting specific axonal arbors and dendritic patterns. Each subtype displays a unique combination of neurochemical markers and firing properties (Gelman and Marin, 2010; Bartolini et al., 2013; Kubota, 2014). Although each inhibitory neuron subtype originates in a distinct region, their overall migration behavior appears similar: in general, immature neurons migrate tangentially over long distances toward the cortex (Nadarajah and Parnavelas, 2002; Tanaka et al., 2003; Lopez-Bendito et al., 2004). They enter the CP from the SVZ, pass through it, and reach the MZ (Tanaka et al., 2009), where they further execute multidirectional tangential migration and become dispersed throughout the cortex (Tanaka et al., 2006, 2009; Inada et al., 2011; Yanagida et al., 2012). In mouse, these neurons settle into their final positions in the $\mathrm{CP}$ postnatally (Hevner et al., 2004; Tanaka et al., 2009).

During tangential migration, inhibitory neurons exhibit a bipolar shape with either an unbranched or branched leading process and a short trailing process. Currently, knowledge of dynamic developmental processes of these neurons is limited. However, Yamasaki et al. (2010) used electroporation with $g f p$ or DsRed plasmid to label cells in the medial ganglionic eminence at E12.5 to assess morphological changes in mouse inhibitory neurons. Perinatally, as those labeled neurons moved from the $\mathrm{MZ}$ to $\mathrm{CP}$, they appeared to transform into a multipolar, "sea urchin"-like shape, exhibiting multiple, thin processes (Yamasaki et al., 2010); no further information relevant to cellular dynamics during this transition is yet available. These processes repeatedly extended and retracted for several hours until one process became unusually long; most of these long processes extended toward the WM, while a minority extended toward the pia. Based on elongation, length and growth dynamics, it is likely that all of these processes represent prospective axons. Currently, it is not known what kind of inhibitory neuron these cells differentiate into; however, the medial ganglionic eminence can produce a variety of inhibitory neurons including Martinotti cells, which extend an axon oriented perpendicular to the pial surface (Gelman and Marin, 2010; Kubota, 2014). The remaining processes of multipolar cells likely become dendrites; however, details of their maturation remain to be elucidated.

It remains uncertain whether these behaviors of inhibitory neurons originating in the medial ganglionic eminence at E12.5 occur in all inhibitory cortical neurons. Nonetheless, these analyses indicate that a subset of inhibitory neurons initiates their polarization from a multipolar cell stage. Future analysis using tools such as genetically-modified mice expressing subtype-specific markers or Cre/CreER drivers (Taniguchi et al., 2011) should determine whether polarity can be established via alternate mechanisms.

\section{Cortical Neurons in situ and Hippocampal Neurons in Dissociation Culture Show Similar Polarity Formation}

The morphological dynamics of neurons undergoing polarization in vitro has been well-studied using time-lapse imaging of hippocampal neurons in dissociated culture (Dotti et al., 1988). After plating, hippocampal neurons typically develop axons and dendrites in five stages (Figure 1): (1) initially round cells form filopodia and lamellipodia; (2) cells extend and retract multiple minor processes; (3) one process transforms into an axon; (4) that axon extends and remaining processes differentiate into dendrites; and (5) differentiated processes mature. The first three stages in particular are key to establishment of polarity.

Evidence indicates that the initial minor processes of cells grown in culture have equal potential to differentiate into an axon: for example, if one experimentally cuts off a process that has grown longer than the others (presumably the future axon), a new potential axon emerges de novo (Dotti and Banker, 1987). Thus, polarity can be definitively established after an axon becomes apparently stable. However, some neurons in vivo are polarized at the time of generation and likely retain some aspects apico-basal polarity of progenitors in the neuroepithelum, or they have front-rear polarity of migrating immature neurons (Hatanaka et al., 2012). Therefore, these neurons in vivo do not need to redefine polarity but rather can inherit aspects of polarity. Indeed, retinal ganglion cells (Zolessi et al., 2006; Randlett et al., 2011) and bipolar cells (Morgan et al., 2006) appear to inherit apicobasal polarity of their progenitors. In retinal ganglion cells, not only the appearance but also the polarized distribution of intracellular components, such as the centrosome and Golgi apparatus, exhibit inheritance of polarity during axon formation (Zolessi et al., 2006; Randlett et al., 2011). Migrating neurons use polarized cellular components to form a leading and a trailing process required for directed movement (Evsyukova et al., 2013). Currently, there are no imaging studies in vivo or in 
situ that directly demonstrate the dynamics of these components during axon formation of migrating neurons. Nonetheless, pontine nucleus neurons form an axon from their leading process (Kawauchi et al., 2006; Watanabe and Murakami, 2009; Shinohara et al., 2013), and trailing processes of cerebellar granule neurons transform into axons (Komuro et al., 2001), indicating that these neurons inherit some elements of front/rear (leading/trailing process) polarity. These mechanisms differ from polarity formation seen in dissociated hippocampal neurons, in which an axon emerges de novo from non-polarized cells.

In contrast, as described above, in both excitatory and inhibitory cortical neurons in vivo or at least in situ, polarity formation primarily occurs in multipolar cells in a manner similar to that seen in dissociated hippocampal neurons. In the next section, we focus on early polarity events that occur during multipolar cell stages prior to axon formation.

\section{Dynamic Processes of Polarity Formation in Excitatory Cortical Neurons}

Radial glial cells, the main progenitor population of excitatory cortical neurons, are neuroepithelial cells that exhibit apicobasal polarity. However, young neurons and intermediate progenitors appear to lose that polarity by retracting apical and basal processes during asymmetric cell division and assuming a multipolar shape (Tabata and Nakajima, 2003; Noctor et al., 2004; Hatanaka and Yamauchi, 2013). Furthermore, intermediate progenitors retract all visible processes and round up prior to division (Miyata et al., 2004; Noctor et al., 2004, 2008), suggesting that they do not inherit the apicobasal polarity exhibited by their progenitors. In addition, multipolar cells do not exhibit stable front-rear polarity, which is seen in actively migrating cells, but instead show highly dynamic behavior, alternately extending and retracting multiple short processes usually $<50 \mu \mathrm{m}$ in length. These cells also show unsteady somal movement (Tabata and Nakajima, 2003; Sakakibara et al., 2014), and some apparently form transient thick processes used to change migration direction (Sakakibara et al., 2014). Random distribution of the centrosome in multipolar cells reported in an imaging study (Sakakibara et al., 2014) and in fixed preparations (Shoukimas and Hinds, 1978) also support the idea that polarity is undetermined at these neuronal stages. After a prolonged period of this activity, a new thin process suddenly emerges and elongates tangentially. Occasionally, a cell retracts that process, even after it reaches $>50 \mu \mathrm{m}$, and extends another (Hatanaka and Yamauchi, 2013), an activity also observed in hippocampal neurons prior to polarity establishment (Dotti et al., 1988). Once a process exceeds $100 \mu \mathrm{m}$, it will likely become an axon and continue to elongate (Hatanaka and Yamauchi, 2013; Namba et al., 2014; Sakakibara et al., 2014). Thus, axon formation of excitatory neurons mostly occurs during the multipolar period while exhibiting unstable or fluctuating polarity.

Recently, several papers have reported that signaling and cytoskeletal proteins function in the multipolar-bipolar transition of excitatory neurons in the IZ (reviewed in Cooper, 2014). To further understand mechanisms governing axon formation in these neurons, it will be important to determine if loss-of-function of those factors merely locks neurons into a multipolar state or also prevents them from forming an axon.

\section{Dynamic Processes of Polarity Formation in Inhibitory Cortical Neurons}

During multidirectional tangential migration in the $\mathrm{MZ}$, inhibitory cortical neurons extend a leading process in the direction of their movement (Tanaka et al., 2009; Inada et al., 2011; Yanagida et al., 2012). After long periods of migration in the MZ (estimated >1d; Tanaka et al., 2009), these neurons descend to the CP (Tanaka et al., 2009). Concomitantly, many transform into multipolar cells that extend numerous short processes, most $<50 \mu \mathrm{m}$ in length, although some are longer (Yamasaki et al., 2010). Because cells in the multipolar stage do not translocate (that is, their soma does not change position significantly), they appear to terminate their migration and lose front-rear polarity. Their short processes repeatedly extend and retract and show no preferential direction of extension. After a prolonged period of this activity, one process abruptly elongates (initial axon formation). As observed in dissociated hippocampal neurons in culture and in excitatory neurons in situ, other processes occasionally extend up to $150-200 \mu \mathrm{m}$ but fail to extend further, and eventually only one exceeds $200 \mu \mathrm{m}$ in length and differentiates into an axon (Yamasaki et al., 2010). Thus, at multipolar stages inhibitory neurons likely do not have fixed polarity, and axon formation occurs in these cells de novo. Further study examining dynamic movement of cellular components in multipolar cells during axon formation should validate this view.

\section{Modes of Polarity Establishment}

There are minor differences between behavior of cortical neurons in situ and hippocampal neurons in vitro. Although in both cases neurons initially appear multipolar, the mode of "random growth and retraction" of processes differs slightly. First, minor processes of hippocampal neurons in vitro show alternate increases and decreases in length, while those of cortical neurons in situ often show alternate appearance and disappearance of processes. Therefore, potential sites of axon initiation seem to be set at the very beginning of the polarization process in vitro. Second, the location of a hippocampal neuron cell body in vitro appears fixed during the multipolar stage, while that of excitatory or inhibitory cortical neurons in situ does not. These activities may be due to microenvironmental differences, such as adhesive properties: hippocampal neurons interact with a positively-charged planar substrate, while cortical neurons do not. Some of the activities one sees in hippocampal neurons in in vitro might be artifacts.

\section{Centrosome Positioning during Polarity Formation}

In vitro and in vivo studies suggest an instructive role for centrosome positioning in axon specification (Lefcort and Bentley, 1989; Zmuda and Rivas, 1998; de Anda et al., 2005, 2010; Andersen and Halloran, 2012). However, recent time-lapse observations of centrosomes in polarizing excitatory cortical neurons in situ reveal that different mechanisms may govern axon formation in these cells (Sakakibara et al., 2014; reviewed in Sakakibara et al., 2013). The centrosome tends to move toward the 
most actively growing process (the so-called "dominant process") and that the initiating axon does not always behave as the dominant process. Neurons undergoing multipolar migration in the IZ form an axon by extending a dominant process toward which the centrosome orients. Thus, the centrosome positions at the base of initiating axon (Sakakibara et al., 2014). Similarly, in polarizing hippocampal neurons in vitro, one minor process becomes an axon and then behaves as the dominant process. In both cases, the centrosome attracted to the growing axon. On the other hand, neurons in the CP at later migration stages exhibit a leading process oriented toward the brain surface, which then behave as the dominant process and attract the centrosome. When an axon forms at the rear of these cells in situ, the centrosome does not translocate toward the initiating axon but rather remains oriented toward the leading process (Sakakibara et al., 2014). Although the latter mode of axon formation may not be primarily observed in vivo, these observations suggest that centrosome positioning is passively controlled by a balance of protrusive activities among processes and does not play an instructive role in excitatory cortical neurons in vivo. In migrating inhibitory cortical neurons, the primary cilium, whose basal body is formed from a centriole, reportedly regulates Sonic hedgehog-mediated reorientation of the leading process (Baudoin et al., 2012). However, the function of the primary cilium in extracellular cue-oriented axonogenesis in these neurons remains unclear. Clarification of the contribution of centrosome/primary cilium to neuronal polarization in vivo may further prompt our understanding of microtubule function underlying axonal morphogenesis.

\section{Cellular Mechanisms Underlying Neuronal Polarization}

Axon specification in cortical neurons is driven by intracellular and extracellular mechanisms (Figure 2). Intracellular signaling molecules relevant to polarization have been identified primarily in in vitro studies of hippocampal neurons, although in vivo studies validating these findings have also been reported. Extracellular mechanisms regulating cortical neuron polarization have been studied by in vivo analyses of knockout phenotypes or gene manipulation in embryonic mouse brain, although most of these studies have been confined to excitatory cortical neurons.

\section{Intracellular Mechanisms}

Cytoskeletal changes dependent on protein phosphorylation are required for axon specification; thus, axon formation can be assessed using markers recognizing differential phosphorylation states of cytoskeletal proteins (Sternberger and Sternberger, 1983; Mandell and Banker, 1996). Stable microtubules within axons confer distinct characteristics based on their organization (Witte et al., 2008; Conde and Cáceres, 2009), and signaling molecules like LKB1 and SAD-A/B kinases trigger axonogenesis by changing the phosphorylation state of microtubule-associated proteins (MAPs), such as Tau and DCX (Kishi et al., 2005; Barnes et al., 2007; Shelly et al., 2007). In excitatory cortical neurons, PKA reportedly activates $\mathrm{LKB} 1$, leading to phosphorylation of
SAD kinases. Consequent downstream signaling of SAD kinases phosphorylates Tau at S262, an event thought to initiate axon formation (Kishi et al., 2005).

The aPKC/Par complex plays a central role in axon specification: for example, aPKC inhibition suppresses axon formation in hippocampal neurons (Zhang et al., 2007). aPKC/Par complex function is differentially regulated by Par3 phosphorylation via multiple kinase pathways (Funahashi et al., 2013; Yang et al., 2014). TGF- $ß$ signaling reportedly increases Par6 phosphorylation, which is required for axon formation by excitatory cortical neurons (Yi et al., 2010). MARKs/Par-1, which acts downstream of the aPKC/Par complex, controls microtubule-binding affinity of DCX (Sapir et al., 2008). The DLK-JNK pathway also regulates DCX phosphorylation and that of other MAPs as well as SCG10/stathmin-2 (Gdalyahu et al., 2004; Eto et al., 2010; Hirai et al., 2011; Westerlund et al., 2011). DOCK7 activation of the small GTPase Rac controls MT stability in axons by inactivation of stathmin/Op18 (Watabe-Uchida et al., 2006). Thus, concerted regulation of microtubule function by multiple kinases and their effectors likely underlies axon formation.

Several small GTPases function differentially in neuronal polarization (Arimura and Kaibuchi, 2007; Gonzalez-Billault et al., 2012). Local activation of the Rap1-Cdc42 pathway has been observed at the tip of an initiating axon in hippocampal neurons in vitro (Schwamborn and Püschel, 2004). Cdc42 reportedly remodels the actin cytoskeleton via cofilin phosphorylation (Garvalov et al., 2007). Rac/Cdc42 also controls retrograde movement of F-actin via phosphorylation of downstream effectors such as PAK1 and Shootin1 (Toriyama et al., 2013). Interestingly, Shootin 1 is implicated in potential crosstalk between the L1-cell adhesion molecule, F-actin, and microtubules in regulating growth cone dynamics during neuronal polarization (Shimada et al., 2008; Sapir et al., 2013), suggesting that coordinated regulation of actin and microtubules is critical for axon formation.

Axon formation also requires directed transport of membrane vesicles and other cargos along polarized microtubules (reviewed in Conde and Cáceres, 2009; Hirokawa et al., 2010; Stiess and Bradke, 2011; Sakakibara et al., 2013). Polarized transport by plus-end-directed motors, such as kinesin-1 (KIF5) and kinesin-2 (KIF3), plays a central role in establishing a single axon (Nakata and Hirokawa, 2003; Jacobson et al., 2006). Identification of several cargo molecules suggests that directed accumulation of signaling and scaffold proteins, such as CRMP-2, PAR-3, and JIP1, is important for axon specification (Nishimura et al., 2004; Kimura et al., 2005; Dajas-Bailador et al., 2008). Localized acetylation of microtubules may regulate cargo/microtubule affinity during axon specification (Reed et al., 2006). PIP $_{3}$ transport by GAKIN/KIF13B functions in axon formation, suggesting a role for accumulated $\mathrm{PIP}_{3}$ in positive feedback regulation of Rac and Cdc42 small GTPases (Horiguchi et al., 2006). Shootin1 also is known as a cargo of Kif20b (Sapir et al., 2013).

Recent studies show that regulators of microtubule dynamics are required for neuronal polarization. Control of microtubule minus-end dynamics by CAMSAP2 is essential for polarization of cortical neurons in vivo (Yau et al., 2014). Altered plus-end dynamics induced by depletion of microtubule regulators, such 


\section{Factors regulating neuronal polarization (axon formation) \\ Cytoskeletal changes \\ Altered microtubule organization and stability (e.g. phosphorylation of MAPs) \\ Actin-mediated extension of protrusions (e.g. activation of small GTPases) \\ Directed transport of membrane vesicles and other cargos \\ Selective transport of polarity regulators toward the prospective axon}

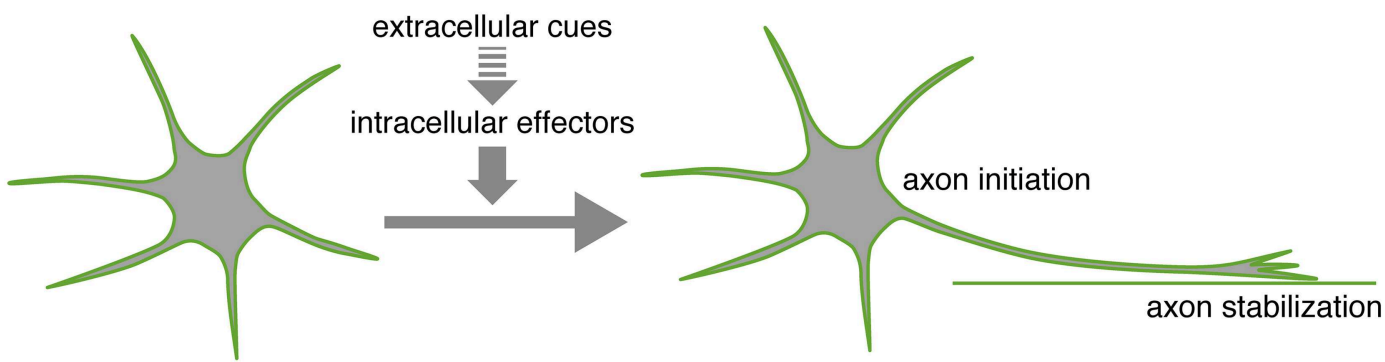

FIGURE 2 | Regulation of cortical neuron polarization. Axon formation in vivo is influenced by extracellular cues. Intracellular effectors regulating cytoskeletal dynamics and membrane vesicle transport function in the initiation, stabilization, and subsequent elongation of a single axon.

as, SLAIN1/2, chTOG/XMAP215, and CLASP2, reportedly underlie polarization defects (Beffert et al., 2012; van der Vaart et al., 2012). These observations suggest that properly controlled microtubule growth, which also underlies microtubuledependent directional transport, is essential to shape axons.

\section{Extracellular Mechanisms}

Interestingly, when the growth cone of an immature process of a hippocampal neuron encounters a preferred substrate in vitro, its growth rapidly increases, while that of other immature processes does not (Esch et al., 1999; Shelly et al., 2007). Observations such as this indicate that external cues also govern polarity formation. Contact with laminin in the basal lamina also triggers retinal ganglion cell axon formation (Zolessi et al., 2006; Randlett et al., 2011). In the case of excitatory cortical neurons, integrity of the surrounding microenvironment may greatly impact axonal specification. Indeed, recent work reveals that these neurons establish directed tangential axon outgrowth due to instructive cues presented on pre-existing efferents: close contact with the cell adhesion molecule TAG-1 on these efferents in the lower IZ stimulates axon formation by multipolar cells, an event mediated in part by downstream Lyn-kinase (Namba et al., 2014). In addition, several extracellular factors, such as the homotypic cell adhesion protein N-cadherin (Gärtner et al., 2012), diffusible protein TGF-ß (Yi et al., 2010) and neurotrophins (Nakamuta et al., 2011), reportedly function as polarization signals for excitatory cortical neurons in vivo. Some investigators propose that a single axon is specified via positive feedback signals that stabilize process extension (Arimura and Kaibuchi, 2007; Inagaki et al., 2011), suggesting that external cues, either contact-mediated or locally diffused, have a stabilizing effect on polarity. In the case of inhibitory neurons, the surrounding environment indeed appears to influence polarity formation: inhibitory neurons do not assume a multipolar shape in dissociated culture. Instead, one of the two processes emerging from these neurons elongates and eventually becomes an axon (Hayashi et al., 2003). Possible cell-cell interactions, such as tiling interactions between neighboring inhibitory neurons, might partially contribute to shape cells in vivo. Because excitatory cortical neurons under the same conditions appear multipolar (Hayashi et al., 2003), intrinsic mechanisms governing axon formation in these two types of neurons may differ despite their similar behavior in vivo.

\section{Concluding Remarks}

Here, we have reviewed recent evidence suggesting that cortical neurons, both excitatory and inhibitory, establish polarity de novo. These neurons initiate axons after assuming a multipolar stage, in which no fixed polarity is exhibited. Although these neurons appear similar during axon formation, it is important, especially in the case of inhibitory neurons, to examine the dynamics of cellular components to validate this view. Also, it will be interesting to examine whether these neuronal subtypes share signaling pathways governing polarity. It should be noted that inhibitory cortical neurons are in fact diverse and consist of multiple morphological subtypes with different spatial and temporal origins. Thus, future investigations are needed to determine whether these subtypes share a common mechanism of axon initiation. In addition, it is important to define dynamic processes governing dendrite formation by both excitatory and inhibitory cortical neurons. These analyses will likely require a combination of genetic labeling of specific excitatory and inhibitory neuronal subtypes with live imaging both in situ and in vivo. 
Finally, analysis of dissociated hippocampal neurons has set the foundation for our current understanding of polarization processes and their molecular basis. Although some of this knowledge is applicable to cortical neurons in situ, care should be taken in generalizing these mechanisms to other neuronal types. Proper understanding of polarity formation in the cerebral cortex requires identification of the key processes that underlie external cue-mediated polarization in vivo.

\section{References}

Andersen, E. F., and Halloran, M. C. (2012). Centrosome movements in vivo correlate with specific neurite formation downstream of LIM homeodomain transcription factor activity. Development 139, 3590-3599. doi: 10.1242/dev.081513

Angevine, J. B. Jr., and Sidman, R. L. (1961). Autoradiographic study of cell migration during histogenesis of cerebral cortex in the mouse. Nature 192, 766-768. doi: $10.1038 / 192766$ b0

Arimura, N., and Kaibuchi, K. (2007). Neuronal polarity: from extracellular signals to intracellular mechanisms. Nat. Rev. Neurosci. 8, 194-205. doi: $10.1038 / \mathrm{nrn} 2056$

Barnes, A. P., Lilley, B. N., Pan, Y. A., Plummer, L. J., Powell, A. W., Raines, A. N., et al. (2007). LKB1 and SAD kinases define a pathway required for the polarization of cortical neurons. Cell 129, 549-563. doi: 10.1016/j.cell.2007.03.025

Barnes, A. P., and Polleux, F. (2009). Establishment of axon-dendrite polarity in developing neurons. Annu. Rev. Neurosci. 32, 347-381. doi: 10.1146/annurev.neuro.31.060407.125536

Bartolini, G., Ciceri, G., and Marin, O. (2013). Integration of GABAergic interneurons into cortical cell assemblies: lessons from embryos and adults. Neuron 79, 849-864. doi: 10.1016/j.neuron.2013.08.014

Baudoin, J. P., Viou, L., Launay, P. S., Luccardini, C., Espeso Gil, S., Kiyasova, V., et al. (2012). Tangentially migrating neurons assemble a primary cilium that promotes their reorientation to the cortical plate. Neuron $76,1108-1122$. doi: 10.1016/j.neuron.2012.10.027

Bayer, S. A., and Altman, J. (1991). Neocortical Development. New York, NY: Raven Press.

Beffert, U., Dillon, G. M., Sullivan, J. M., Stuart, C. E., Gilbert, J. P., Kambouris, J. A., et al. (2012). Microtubule plus-end tracking protein CLASP2 regulates neuronal polarity and synaptic function. J. Neurosci. 32, 13906-13916. doi: 10.1523/JNEUROSCI.2108-12.2012

Ceci, M. L., Pedraza, M., and de Carlos, J. A. (2012). The embryonic septum and ventral pallium, new sources of olfactory cortex cells. PLOS ONE 7:e44716. doi: 10.1371/journal.pone.0044716

Conde, C., and Cáceres, A. (2009). Microtubule assembly, organization and dynamics in axons and dendrites. Nat. Rev. Neurosci. 10, 319-332. doi: $10.1038 / \mathrm{nrn} 2631$

Cooper, J. A. (2014). Molecules and mechanisms that regulate multipolar migration in the intermediate zone. Front. Cell. Neurosci. 8:386. doi: $10.3389 /$ fncel.2014.00386

Dajas-Bailador, F., Jones, E. V., and Whitmarsh, A. J. (2008). The JIP1 scaffold protein regulates axonal development in cortical neurons. Curr. Biol. 18, 221-226. doi: 10.1016/j.cub.2008.01.025

de Anda, F. C., Meletis, K., Ge, X., Rei, D., and Tsai, L.-H. (2010). Centrosome motility is essential for initial axon formation in the neocortex. J. Neurosci. 30, 10391-10406. doi: 10.1523/JNEUROSCI.0381-10.2010

de Anda, F. C., Pollarolo, G., Da Silva, J. S., Camoletto, P. G., Feiguin, F., and Dotti, C. G. (2005). Centrosome localization determines neuronal polarity. Nature 436, 704-708. doi: 10.1038/nature03811

de Carlos, J. A., Lopez-Mascaraque, L., and Valverde, F. (1996). Dynamics of cell migration from the lateral ganglionic eminence in the rat. J. Neurosci. 16, 6146-6156.

Dotti, C. G., and Banker, G. A. (1987). Experimentally induced alteration in the polarity of developing neurons. Nature 330, 254-256. doi: 10.1038/330254a0

Dotti, C. G., Sullivan, C. A., and Banker, G. A. (1988). The establishment of polarity by hippocampal neurons in culture. J. Neurosci. 8, 1454-1468.

\section{Acknowledgments}

We thank Drs. Tamar Sapir, Kenta Yamauchi, Masashi Kishi, and Yasuo Kawaguchi for critical reading of the manuscript. AS was supported by JSPS KAKENHI $(23500410,26640040)$ and the Narishige Zoological Science Award. YH was supported by JSPS KAKENHI $(22500310,25430020)$ and MEXT KAKENHI (25123722).

Esch, T., Lemmon, V., and Banker, G. (1999). Local presentation of substrate molecules directs axon specification by cultured hippocampal neurons. J. Neurosci. 19, 6417-6426.

Eto, K., Kawauchi, T., Osawa, M., Tabata, H., and Nakajima, K. (2010). Role of dual leucine zipper-bearing kinase (DLK/MUK/ZPK) in axonal growth. Neurosci. Res. 66, 37-45. doi: 10.1016/j.neures.2009.09.1708

Evsyukova, I., Plestant, C., and Anton, E. S. (2013). Integrative mechanisms of oriented neuronal migration in the developing brain. Annu. Rev. Cell Dev. Biol. 29, 299-353. doi: 10.1146/annurev-cellbio-101512-122400

Funahashi, Y., Namba, T., Fujisue, S., Itoh, N., Nakamuta, S., Kato, K., et al. (2013). ERK2-mediated phosphorylation of Par3 regulates neuronal polarization. J. Neurosci. 33, 13270-13285. doi: 10.1523/JNEUROSCI.4210-12.2013

Gärtner, A., Fornasiero, E. F., Munck, S., Vennekens, K., Seuntjens, E., Huttner, W. B., et al. (2012). N-cadherin specifies first asymmetry in developing neurons. EMBO J. 31, 1893-1903. doi: 10.1038/emboj.2012.41

Garvalov, B. K., Flynn, K. C., Neukirchen, D., Meyn, L., Teusch, N., Wu, X., et al. (2007). Cdc42 regulates cofilin during the establishment of neuronal polarity. J. Neurosci. 27, 13117-13129. doi: 10.1523/JNEUROSCI.3322-07.2007

Gdalyahu, A., Ghosh, I., Levy, T., Sapir, T., Sapoznik, S., Fishler, Y., et al. (2004). DCX, a new mediator of the JNK pathway. EMBO J. 23, 823-832. doi: 10.1038/sj.emboj.7600079

Gelman, D. M., and Marin, O. (2010). Generation of interneuron diversity in the mouse cerebral cortex. Eur. J. Neurosci. 31, 2136-2141. doi: 10.1111/j.14609568.2010.07267.x

Gonzalez-Billault, C., Munoz-Llancao, P., Henriquez, D. R., Wojnacki, J., Conde, C., and Caceres, A. (2012). The role of small GTPases in neuronal morphogenesis and polarity. Cytoskeleton (Hoboken) 69, 464-485. doi: 10.1002/cm. 21034

Greig, L. C., Woodworth, M. B., Galazo, M. J., Padmanabhan, H., and Macklis, J. D. (2013). Molecular logic of neocortical projection neuron specification, development and diversity. Nat. Rev. Neurosci. 14, 755-769. doi: 10.1038/nrn3586

Hatanaka, Y., and Murakami, F. (2002). In vitro analysis of the origin, migratory behavior, and maturation of cortical pyramidal cells. J. Comp. Neurol. 454, 1-14. doi: 10.1002/cne.10421

Hatanaka, Y., and Yamauchi, K. (2013). Excitatory cortical neurons with multipolar shape establish neuronal polarity by forming a tangentially oriented axon in the intermediate zone. Cereb. Cortex 23, 105-113. doi: 10.1093/cercor/ bhr383

Hatanaka, Y., Yamauchi, K., and Murakami, F. (2012). Formation of axon-dendrite polarity in situ: initiation of axons from polarized and non-polarized cells. Dev. Growth Differ. 54, 398-407. doi: 10.1111/j.1440-169X.2012.01344.x

Hayashi, K., Kawai-Hirai, R., Harada, A., and Takata, K. (2003). Inhibitory neurons from fetal rat cerebral cortex exert delayed axon formation and active migration in vitro. J. Cell Sci. 116, 4419-4428. doi: 10.1242/jcs.00762

Hevner, R. F., Daza, R. A., Englund, C., Kohtz, J., and Fink, A. (2004). Postnatal shifts of interneuron position in the neocortex of normal and reeler mice: evidence for inward radial migration. Neuroscience 124, 605-618. doi: 10.1016/j.neuroscience.2003.11.033

Hirai, S., Banba, Y., Satake, T., and Ohno, S. (2011). Axon formation in neocortical neurons depends on stage-specific regulation of microtubule stability by the dual leucine zipper kinase-c-Jun N-terminal kinase pathway. J. Neurosci. 31, 6468-6480. doi: 10.1523/JNEUROSCI.5038-10.2011

Hirokawa, N., Niwa, S., and Tanaka, Y. (2010). Molecular motors in neurons: transport mechanisms and roles in brain function, development, and disease. Neuron 68, 610-638. doi: 10.1016/j.neuron.2010.09.039 
Horiguchi, K., Hanada, T., Fukui, Y., and Chishti, A. H. (2006). Transport of $\mathrm{PIP}_{3}$ by GAKIN, a kinesin-3 family protein, regulates neuronal cell polarity. J. Cell Biol. 174, 425-436. doi: 10.1083/jcb.200604031

Inada, H., Watanabe, M., Uchida, T., Ishibashi, H., Wake, H., Nemoto, T., et al. (2011). GABA regulates the multidirectional tangential migration of GABAergic interneurons in living neonatal mice. PLoS ONE 6:e27048. doi: 10.1371/journal.pone. 0027048

Inagaki, N., Toriyama, M., and Sakumura, Y. (2011). Systems biology of symmetry breaking during neuronal polarity formation. Dev. Neurobiol. 71, 584-593. doi: $10.1002 /$ dneu.20837

Jacobson, C., Schnapp, B., and Banker, G. A. (2006). A change in the selective translocation of the Kinesin-1 motor domain marks the initial specification of the axon. Neuron 49, 797-804. doi: 10.1016/j.neuron.2006.02.005

Jones, E. G. (1984). "Laminar distribution of cortical efferent cells," in Cerebral Cortex, Vol. 1, Cellular Components of the Cerebral Cortex, eds A. Peters and E. G. Jones (New York, NY: Plenum Press), 521-553.

Kawaguchi, Y. (1993). Groupings of nonpyramidal and pyramidal cells with specific physiological and morphological characteristics in rat frontal cortex. J. Neurophysiol. 69, 416-431.

Kawauchi, D., Taniguchi, H., Watanabe, H., Saito, T., and Murakami, F. (2006). Direct visualization of nucleogenesis by precerebellar neurons: involvement of ventricle-directed, radial fibre-associated migration. Development 133, 1113-1123. doi: 10.1242/dev.02283

Kimura, T., Arimura, N., Fukata, Y., Watanabe, H., Iwamatsu, A., and Kaibuchi, K. (2005). Tubulin and CRMP-2 complex is transported via Kinesin-1. J. Neurochem. 93, 1371-1382. doi: 10.1111/j.1471-4159.2005.03063.x

Kishi, M., Pan, Y. A., Crump, J. G., and Sanes, J. R. (2005). Mammalian SAD kinases are required for neuronal polarization. Science 307, 929-932. doi: 10.1126/science. 1107403

Komuro, H., Yacubova, E., Yacubova, E., and Rakic, P. (2001). Mode and tempo of tangential cell migration in the cerebellar external granular layer. J. Neurosci. $21,527-540$.

Kubota, Y. (2014). Untangling GABAergic wiring in the cortical microcircuit. Curr. Opin. Neurobiol. 26, 7-14. doi: 10.1016/j.conb.2013.10.003

Lefcort, F., and Bentley, D. (1989). Organization of cytoskeletal elements and organelles preceding growth cone emergence from an identified neuron in situ. J. Cell Biol. 108, 1737-1749. doi: 10.1083/jcb.108.5.1737

Lopez-Bendito, G., Sturgess, K., Erdelyi, F., Szabo, G., Molnar, Z., and Paulsen, O. (2004). Preferential origin and layer destination of GAD65-GFP cortical interneurons. Cereb. Cortex 14, 1122-1133. doi: 10.1093/cercor/bhh072

Mandell, J. W., and Banker, G. A. (1996). A spatial gradient of tau protein phosphorylation in nascent axons. J. Neurosci. 16, 5727-5740.

Miyata, T., Kawaguchi, A., Okano, H., and Ogawa, M. (2001). Asymmetric inheritance of radial glial fibers by cortical neurons. Neuron 31, 727-741. doi: 10.1016/S0896-6273(01)00420-2

Miyata, T., Kawaguchi, A., Saito, K., Kawano, M., Muto, T., and Ogawa, M. (2004). Asymmetric production of surface-dividing and non-surface-dividing cortical progenitor cells. Development 131, 3133-3145. doi: 10.1242/dev.01173

Molyneaux, B. J., Arlotta, P., Menezes, J. R., and Macklis, J. D. (2007). Neuronal subtype specification in the cerebral cortex. Nat. Rev. Neurosci. 8, 427-437. doi: $10.1038 / \mathrm{nrn} 2151$

Morgan, J. L., Dhingra, A., Vardi, N., and Wong, R. O. (2006). Axons and dendrites originate from neuroepithelial-like processes of retinal bipolar cells. Nat. Neurosci. 9, 85-92. doi: 10.1038/nn1615

Nadarajah, B., and Parnavelas, J. G. (2002). Modes of neuronal migration in the developing cerebral cortex. Nat. Rev. Neurosci. 3, 423-432. doi: 10.1038/nrn845

Nakamuta, S., Funahashi, Y., Namba, T., Arimura, N., Picciotto, M. R., Tokumitsu, H., et al. (2011). Local application of neurotrophins specifies axons through inositol 1,4,5-trisphosphate, calcium, and Ca2+/calmodulin-dependent protein kinases. Sci. Signal. 4, ra76. doi: 10.1126/scisignal.2002011

Nakata, T., and Hirokawa, N. (2003). Microtubules provide directional cues for polarized axonal transport through interaction with kinesin motor head. J. Cell Biol. 162, 1045-1055. doi: 10.1083/jcb.200302175

Namba, T., Kibe, Y., Funahashi, Y., Nakamuta, S., Takano, T., Ueno, T., et al. (2014). Pioneering axons regulate neuronal polarization in the developing cerebral cortex. Neuron 81, 814-829. doi: 10.1016/j.neuron.2013.12.015

Nishimura, T., Kato, K., Yamaguchi, T., Fukata, Y., Ohno, S., and Kaibuchi, K. (2004). Role of the PAR-3-KIF3 complex in the establishment of neuronal polarity. Nat. Cell Biol. 6, 328-334. doi: 10.1038/ncb1118
Noctor, S. C., Flint, A. C., Weissman, T. A., Dammerman, R. S., and Kriegstein, A. R. (2001). Neurons derived from radial glial cells establish radial units in neocortex. Nature 409, 714-720. doi: 10.1038/35055553

Noctor, S. C., Martinez-Cerdeno, V., Ivic, L., and Kriegstein, A. R. (2004). Cortical neurons arise in symmetric and asymmetric division zones and migrate through specific phases. Nat. Neurosci. 7, 136-144. doi: 10.1038/nn1172

Noctor, S. C., Martinez-Cerdeno, V., and Kriegstein, A. R. (2008). Distinct behaviors of neural stem and progenitor cells underlie cortical neurogenesis. J. Comp. Neurol. 508, 28-44. doi: 10.1002/cne.21669

Pontious, A., Kowalczyk, T., Englund, C., and Hevner, R. F. (2008). Role of intermediate progenitor cells in cerebral cortex development. Dev. Neurosci. 30, 24-32. doi: 10.1159/000109848

Rakic, P. (1972). Mode of cell migration to the superficial layers of fetal monkey neocortex. J. Comp. Neurol. 145, 61-83. doi: 10.1002/cne.901450105

Rakic, P., Knyihar-Csillik, E., and Csillik, B. (1996). Polarity of microtubule assemblies during neuronal cell migration. Proc. Natl. Acad. Sci. U.S.A. 93, 9218-9222. doi: 10.1073/pnas.93.17.9218

Randlett, O., Poggi, L., Zolessi, F. R., and Harris, W. A. (2011). The oriented emergence of axons from retinal ganglion cells is directed by laminin contact in vivo. Neuron 70, 266-280. doi: 10.1016/j.neuron.2011.03.013

Reed, N. A., Cai, D., Blasius, T. L., Jih, G. T., Meyhofer, E., Gaertig, J., et al. (2006). Microtubule acetylation promotes kinesin-1 binding and transport. Curr. Biol. 16, 2166-2172. doi: 10.1016/j.cub.2006.09.014

Sakakibara, A., Ando, R., Sapir, T., and Tanaka, T. (2013). Microtubule dynamics in neuronal morphogenesis. Open Biol. 3:130061. doi: 10.1098/rsob.130061

Sakakibara, A., Sato, T., Ando, R., Noguchi, N., Masaoka, M., and Miyata, T. (2014). Dynamics of centrosome translocation and microtubule organization in neocortical neurons during distinct modes of polarization. Cereb. Cortex 24, 1301-1310. doi: 10.1093/cercor/bhs411

Sapir, T., Levy, T., Sakakibara, A., Rabinkov, A., Miyata, T., and Reiner, O. (2013). Shootin1 acts in concert with KIF20B to promote polarization of migrating neurons. J. Neurosci. 33, 11932-11948. doi: 10.1523/JNEUROSCI.5425-12.2013

Sapir, T., Shmueli, A., Levy, T., Timm, T., Elbaum, M., Mandelkow, E. M., et al. (2008). Antagonistic effects of doublecortin and MARK2/Par1 in the developing cerebral cortex. J. Neurosci. 28, 13008-13013. doi: 10.1523/JNEUROSCI.2363-08.2008

Schwamborn, J. C., and Püschel, A. W. (2004). The sequential activity of the GTPases Rap1B and Cdc42 determines neuronal polarity. Nat. Neurosci. 7, 923-929. doi: 10.1038/nn1295

Shelly, M., Cancedda, L., Heilshorn, S., Sumbre, G., and Poo, M. M. (2007). LKB1/STRAD promotes axon initiation during neuronal polarization. Cell 129, 565-577. doi: 10.1016/j.cell.2007.04.012

Shimada, T., Toriyama, M., Uemura, K., Kamiguchi, H., Sugiura, T., Watanabe, N., et al. (2008). Shootin1 interacts with actin retrograde flow and L1-CAM to promote axon outgrowth. J. Cell Biol. 181, 817-829. doi: 10.1083/jcb.200712138

Shinohara, M., Zhu, Y., and Murakami, F. (2013). Four-dimensional analysis of nucleogenesis of the pontine nucleus in the hindbrain. J. Comp. Neurol. 521, 3340-3357. doi: 10.1002/cne.23353

Shoukimas, G. M., and Hinds, J. W. (1978). The development of the cerebral cortex in the embryonic mouse: an electron microscopic serial section analysis. J. Comp. Neurol. 179, 795-830. doi: 10.1002/cne.901790407

Sternberger, L. A., and Sternberger, N. H. (1983). Monoclonal antibodies distinguish phosphorylated and nonphosphorylated forms of neurofilaments in situ. Proc. Natl. Acad. Sci. U.S.A. 80, 6126-6130. doi: 10.1073/pnas.80.19.6126

Stiess, M., and Bradke, F. (2011). Neuronal polarization: the cytoskeleton leads the way. Dev. Neurobiol. 71, 430-444. doi: 10.1002/dneu.20849

Tabata, H., and Nakajima, K. (2003). Multipolar migration: the third mode of radial neuronal migration in the developing cerebral cortex. J. Neurosci. 23, 9996-10001.

Tanaka, D., Nakaya, Y., Yanagawa, Y., Obata, K., and Murakami, F. (2003). Multimodal tangential migration of neocortical GABAergic neurons independent of GPI-anchored proteins. Development 130, 5803-5813. doi: 10.1242/dev. 00825

Tanaka, D. H., Maekawa, K., Yanagawa, Y., Obata, K., and Murakami, F. (2006). Multidirectional and multizonal tangential migration of GABAergic interneurons in the developing cerebral cortex. Development 133, 2167-2176. doi: 10.1242/dev.02382

Tanaka, D. H., Yanagida, M., Zhu, Y., Mikami, S., Nagasawa, T., Miyazaki, J., et al. (2009). Random walk behavior of migrating cortical interneurons in 
the marginal zone: time-lapse analysis in flat-mount cortex. J. Neurosci. 29, 1300-1311. doi: 10.1523/JNEUROSCI.5446-08.2009

Taniguchi, H., He, M., Wu, P., Kim, S., Paik, R., Sugino, K., et al. (2011). A resource of Cre driver lines for genetic targeting of GABAergic neurons in cerebral cortex. Neuron 71, 995-1013. doi: 10.1016/j.neuron.2011.07.026

Toriyama, M., Kozawa, S., Sakumura, Y., and Inagaki, N. (2013). Conversion of a signal into forces for axon outgrowth through Pak1-mediated shootin1 phosphorylation. Curr. Biol. 23, 529-534. doi: 10.1016/j.cub.2013.02.017

van der Vaart, B., Franker, M. A., Kuijpers, M., Hua, S., Bouchet, B. P., Jiang, K., et al. (2012). Microtubule plus-end tracking proteins SLAIN1/2 and ch-TOG promote axonal development. J. Neurosci. 32, 14722-14728. doi: 10.1523/JNEUROSCI.1240-12.2012

Watabe-Uchida, M., John, K. A., Janas, J. A., Newey, S. E., and Van Aelst, L. (2006). The Rac activator DOCK7 regulates neuronal polarity through local phosphorylation of stathmin/Op18. Neuron 51, 727-739. doi: 10.1016/j.neuron.2006.07.020

Watanabe, H., and Murakami, F. (2009). Real time analysis of pontine neurons during initial stages of nucleogenesis. Neurosci. Res. 64, 20-29. doi: 10.1016/j.neures.2009.01.007

Westerlund, N., Zdrojewska, J., Padzik, A., Komulainen, E., Björkblom, B., Rannikko, E., et al. (2011). Phosphorylation of SCG10/stathmin-2 determines multipolar stage exit and neuronal migration rate. Nat. Neurosci. 14, 305-313. doi: $10.1038 / \mathrm{nn} .2755$

Witte, H., Neukirchen, D., and Bradke, F. (2008). Microtubule stabilization specifies initial neuronal polarization. J. Cell Biol. 180, 619-632. doi: 10.1083/jcb.200707042

Yamasaki, E., Tanaka, D. H., Yanagawa, Y., and Murakami, F. (2010). Cortical GABAergic interneurons transiently assume a sea urchin-like nonpolarized shape before axon initiation. J. Neurosci. 30, 15221-15227. doi: 10.1523/JNEUROSCI.1527-10.2010

Yanagida, M., Miyoshi, R., Toyokuni, R., Zhu, Y., and Murakami, F. (2012). Dynamics of the leading process, nucleus, and Golgi apparatus of migrating cortical interneurons in living mouse embryos. Proc. Natl. Acad. Sci. U.S.A. 109, 16737-16742. doi: 10.1073/pnas.1209166109

Yang, R., Kong, E., Jin, J., Hergovich, A., and Püschel, A. W. (2014). Rassf5 and $\mathrm{Ndr}$ kinases regulate neuronal polarity through Par3 phosphorylation in a novel pathway. J. Cell Sci. 127, 3463-3476. doi: 10.1242/jcs.146696

Yau, K. W., van Beuningen, S. F., Cunha-Ferreira, I., Cloin, B. M., van Battum, E. Y., Will, L., et al. (2014). Microtubule minus-end binding protein CAMSAP2 controls axon specification and dendrite development. Neuron 82, 1058-1073. doi: 10.1016/j.neuron.2014.04.019

Yi, J. J., Barnes, A. P., Hand, R., Polleux, F., and Ehlers, M. D. (2010). TGF-beta signaling specifies axons during brain development. Cell 142, 144-157. doi: 10.1016/j.cell.2010.06.010

Zhang, X., Zhu, J., Yang, G. Y., Wang, Q. J., Qian, L., Chen, Y. M., et al. (2007) Dishevelled promotes axon differentiation by regulating atypical protein kinase C. Nat. Cell Biol. 9, 743-754. doi: 10.1038/ncb1603

Zmuda, J. F., and Rivas, R. J. (1998). The Golgi apparatus and the centrosome are localized to the sites of newly emerging axons in cerebellar granule neurons in vitro. Cell Motil. Cytoskeleton 41, 18-38.

Zolessi, F. R., Poggi, L., Wilkinson, C. J., Chien, C. B., and Harris, W. A. (2006). Polarization and orientation of retinal ganglion cells in vivo. Neural Dev. 1:2. doi: 10.1186/1749-8104-1-2

Conflict of Interest Statement: The authors declare that the research was conducted in the absence of any commercial or financial relationships that could be construed as a potential conflict of interest.

Copyright (c) 2015 Sakakibara and Hatanaka. This is an open-access article distributed under the terms of the Creative Commons Attribution License (CC BY). The use, distribution or reproduction in other forums is permitted, provided the original author(s) or licensor are credited and that the original publication in this journal is cited, in accordance with accepted academic practice. No use, distribution or reproduction is permitted which does not comply with these terms. 